\title{
Colaboración de un centro sociosanitario y dos farmacias comunitarias en la revisión de la medicación y elaboración de sistemas personalizados de dosificación
}

\author{
Fernando Mud Castelló', Sara Mud Castelló2 ${ }^{2}$ María José Rodríguez Moncho², Almudena Signes Mut' \\ Pasqual Muñoz Ballester ${ }^{3}$ \\ 1. Farmacéutico comunitario. Farmacia Fernando Mud (Ondara). 2. Farmacéutica comunitaria. Farmacia M. Dolores Castelló (Ondara). 3. Médico \\ residencia Nuestra Señora de la Soledad (Ondara).
}

\section{PALABRAS CLAVE}

Farmacia comunitaria, criterios STOPP-START, SPD, revisión de medicamentos

\section{ABREVIATURAS}

OMS: Organización Mundial de la Salud

PPI: prescripciones potencialmente inapropiadas

PPO: prescripciones potencialmente omitidas

SPD: sistema personalizado de dosificación de medicamentos V2: versión 2

\section{RESUMEN}

Introducción: Los pacientes institucionalizados tienen, por sus características, más posibilidades de presentar problemas de seguridad con los medicamentos. La farmacia comunitaria puede ofrecer diferentes servicios, como es la revisión de la medicación. Para ello, contamos con los criterios STOPP, que detectan prescripciones potencialmente inapropiadas (PPI) y los START que detectan prescripciones potencialmente omitidas (PP0). Además de la revisión de la medicación, el farmacéutico comunitario dispone de los sistemas personalizados de dosificación (SPD).

Objetivo: Facilitar la adherencia a la medicación prescrita en un centro sociosanitario con ayuda de los SPD realizados por los farmacéuticos comunitarios.

Metodología: Estudio prospectivo realizado en dos farmacias comunitarias y en un centro sociosanitario (25 residentes) de Ondara (Alicante) durante ocho meses (enero-agosto 2017). En él participaron un médico, un enfermero y cuatro farmacéuticos comunitarios.

Resultados: Se incluyeron 31 pacientes que generaron 62 revisiones de la medicación. La edad media fue de 83 años. Se detectaron 16 PPI y el médico aceptó el 50\% de las intervenciones. El criterio STOPP más detectado fue el B9: diuréticos de asa para el tratamiento de la hipertensión con incontinencia urinaria.

Se detectaron 25 criterios PPO y el facultativo aceptó el 28\% de las intervenciones. El criterio START más detectado fue el E4: antirresortivos o anabolizantes óseos en pacientes con osteopororosis.

Conclusiones: Se ha facilitado la administración de la medicación en el centro sociosanitario desde que se realizan los SPD por parte de los farmacéuticos comunitarios. Con la revisión de la medicación el farmacéutico comunitario ayudó al médico a optimizar la farmacoterapia de los pacientes ingresados.

Collaboration of a health and social services center and community pharmacies in reviewing medications and processing personalized dosing systems

\section{ABSTRACT \\ Introduction: Institutionalized patients have, by their characteristics, more possibili- ties of presenting security problems with medication. The community pharmacy can offer different services, such as medication review. For this, we have the STOPP criteria, which detect potentially inappropriate prescriptions (PPI) and the START that detect potentially omitted prescriptions (PPO). In addition to the medication review, the community phar- macist has personalized dispensation systems (SPD).}

Este trabajo obtuvo el premio a la mejor experiencia en la implantación del SPD en una farmacia para la mejora de la adherencia terapéutica mediante el uso del Servicio Personalizado de Dosificación (SPD) en farmacias comunitarias españolas otorgado por la Fundación Mylan y SEFAC.

Recibido: $16 / 4 / 2018$ Aceptado: 19/5/2018

Disponible online: 30-6-2018
Financiación: ninguna.

Conflicto de intereses: ninguno.

Contribución a la autoría: todos los autores participaron por igual en el diseño y desarrollo del estudio, análisis de los resultados, redacción del manuscrito y revisión del contenido final.

Cite este artículo como: Mud F, Mud S, Rodríguez MJ, Signes A, Muñoz P. Colaboración de un centro sociosanitario y dos farmacias comunitarias en la revisión de la medicación y elaboración de sistemas personalizados de dosificación. Farmacéuticos Comunitarios. 2018 Jun 30; 10(2):15-20. doi:10.5672/FC.2173-9218.(2018/Vol10).002.03

Correspondencia: Fernando Mud Castelló (fernando@farmaondara.es)

ISSN 1885-8619 @ SEFAC (Sociedad Española de Farmacia Familiar y Comunitaria). Todos los derechos reservados. 


\section{KEYWORDS}

Community pharmacy, STOPP-START criteria, SPD, medication review
Objective: Facilitate adherence to the prescribed medications in a social-health center with the help of the SPD carried out by community pharmacists.

Methodology: Prospective study carried out in two community pharmacies and in a social health center (25 residents) of Ondara for eight months (January-August 2017). It involved a doctor, a nurse and four community pharmacists.

Results: 31 patients were included, which generated 62 reviews of the medication. The average age was 83 years. 16 PPI were detected and the doctor accepted 50\% of them. The most detected STOPP criterion was B9: loop diuretics for the treatment of hypertension with urinary incontinence.

25 PPO criteria were detected and the doctor accepted $28 \%$ of them. The most detected START criterion was E4: Antiresorptive or bone anabolic agents in patients with osteopororosis.

Conclusions: The administration of medication in the social-health center has been facilitated since the MDSs were carried out by the community pharmacists. With the review of the medication the community pharmacist helped the doctor to optimize the pharmacotherapy of admitted patients.

\section{Introducción}

Las personas mayores son un grupo heterogéneo en el que generalmente coexisten diversas patologías para las que toman varios medicamentos. La polimedicación incrementa la posibilidad de que aparezcan problemas relacionados con la medicación tales como interacciones medicamentosas o efectos no deseados. También dificulta la administración de los fármacos por el propio paciente o persona encargada de su manejo en los centros sociosanitarios.

Para minimizar estos riesgos se necesita una atención integral del paciente a través de la actuación sinérgica de los servicios sanitarios y sociales para garantizar la seguridad del paciente. Definimos la seguridad del paciente como el conjunto de actuaciones orientadas a evitar, prevenir o minimizar el daño producido al paciente como resultado de los cuidados de salud prestados.

En el caso de la farmacoterapia es muy importante la adherencia a los tratamientos prescritos por el médico. La Organización Mundial de la Salud (OMS) definió la adherencia terapéutica como el grado en el que la conducta del paciente en relación a la toma de medicación y/o seguimiento de hábitos saludables se corresponde con las recomendaciones acordadas con el profesional sanitario [1].

En los países desarrollados solo el 50\% de los pacientes crónicos cumple con su tratamiento [1]. Esto supone un problema grave de salud, porque además de las repercusiones negativas a nivel clínico también las hay a nivel económico, de ahí la importancia de establecer estrategias dirigidas a mejorar la adherencia.
Por otra parte, es necesario mejorar la calidad de vida de las personas mayores. Existen los centros sociosanitarios tipo residencia para mayores dependientes, muchos de ellos con deterioro cognitivo. Se trata de centros con asistencia sanitaria limitada, generalmente con pocos recursos, que se apoyan parcialmente en el Sistema Nacional de Salud. Los pacientes institucionalizados tienen, por sus características, más posibilidades de presentar problemas de seguridad con los medicamentos [2,3].

Entre las actuaciones que se pueden realizar en estos centros para minimizar los problemas con la medicación están: mejorar la organización de los centros para repartir el trabajo, usar sistemas adecuados de administración de los medicamentos, prescripción adecuada a la población geriátrica, revisión periódica de tratamientos, la conveniencia de un farmacéutico para la preparación, distribución y supervisión de los tratamientos. Con todo esto, podemos decir que es necesaria una intervención conjunta de los diferentes profesionales del sistema sanitario para garantizar en la medida de lo posible la seguridad del paciente.

Existen diferentes servicios que se pueden prestar desde la farmacia comunitaria para mejorar la seguridad del paciente.

Uno de los servicios que se pueden ofrecer es la revisión de la medicación. Para ello, contamos con los criterios STOPP-START. Se trata de criterios basados en la evidencia organizados por sistemas, que pueden aplicarse fácilmente, y que recogen los errores más comunes de tratamiento y omisión en la prescripción. Los criterios STOPP son una herra- mienta para detectar prescripciones potencialmente inapropiadas (PPI) en ancianos, mientras que los START sirven para detectar tratamientos indicados y apropiados en personas mayores, omitidos siempre que padezcan determinadas enfermedades y no tengan ninguna contraindicación [4]. Además de la revisión de la medicación, el farmacéutico comunitario dispone de los sistemas personalizados de dosificación (SPD). Son una herramienta que ayuda a reducir los problemas asociados a la medicación y a mejorar la adherencia al tratamiento, así como facilitar la administración de los medicamentos en los centros sociosanitarios. El SPD es un envase cerrado en el que el farmacéutico reacondiciona la medicación semanal del paciente, separada en días y tomas, previa revisión de la misma.

\section{Objetivos}

Objetivo principal del estudio

- Facilitar la adherencia a la medicación prescrita en un centro sociosanitario con ayuda de los SPD realizados por los farmacéuticos comunitarios.

\section{Objetivos secundarios}

- Integrar a los farmacéuticos comunitarios en el equipo médico del centro sociosanitario.

- Revisar la medicación y detectar los fallos en la aplicación de los criterios STOPP-START versión 2 (V2) para mejorar la seguridad del paciente.

- Analizar la aceptación de los criterios STOPP-START V2 por parte del médico del centro sociosanitario. 


\section{Métodos}

Estudio prospectivo realizado en dos farmacias comunitarias y en un centro sociosanitario (25 residentes) de Ondara durante ocho meses (enero-agosto 2017). En él participaron un médico, un enfermero y cuatro farmacéuticos comunitarios.

Para poder formar parte del estudio, a todos los pacientes o familiares se les explicaba el proyecto y se les entregaba un documento informativo. Todos aquellos que aceptaron participar firmaron un documento de consentimiento informado.

Los criterios de inclusión fueron:

- Pacientes ingresados en el centro sociosanitario y que firmaban el consentimiento informado.

- Al menos un medicamento de la farmacoterapia tenía que ser acondicionable para usar en SPD.

Los criterios de exclusión fueron:

- Pacientes sin ningún fármaco acondicionable para usar en SPD.

\section{Procedimiento}

Fue elaborado por los diferentes profesionales sanitarios que participaron.

1. Alta del paciente en el programa: el facultativo rellenaba la ficha del paciente con los datos sociodemográficos (nombre, edad, número tarjeta sanitaria, DNI) diagnósticos médicos y tratamientos (principio activo, dosis, forma farmacéutica y posología).

2. Revisión de la medicación por parte del farmacéutico comunitario: a partir de esta ficha los farmacéuticos revisaban el tratamiento siguiendo los criterios STOPP-START V2 [4].

3. Intervención: los resultados obtenidos eran comunicados al médico que respondía si aceptaba la propuesta del farmacéutico comunitario o no. En ambos casos el farmacéutico registraba la intervención que realizaba el médico.

Esto se realizaba inicialmente a todos los pacientes nuevos (visita inicial) y después en las sucesivas modificaciones del tratamiento (visita continuación).

Si el médico aceptaba el criterio STOPP-START detectado por el farmacéutico comunitario, generaba una nueva ficha de paciente $y$, por tanto, una nueva visita de intervención.
Cuando el facultativo no aceptaba el criterio STOPP-START, justificando el motivo, se aprobaba el tratamiento y no se generaba una nueva visita de intervención.

En el presente estudio, si en una visita de continuación se detectaba un criterio STOPP-START que el médico ya había rechazado anteriormente, no se contabilizaba como un nuevo criterio.

Las causas de no aceptación de alguno de los criterios que se definieron fueron las siguientes:

- Paciente controlado.

- Intolerancia al medicamento propuesto.

- Diagnostico incorrecto.

- Necesidad del medicamento porque otras alternativas no tuvieron efecto.

4. Realización del SPD: una vez revisado el tratamiento se procedía a realizar los SPD. Para ello se comprobaba la ficha técnica del medicamento a través del programa informático Bot Plus para determinar si los medicamentos eran acondicionables o no para usar en SPD. Cuando no lo eran se intentaba buscar alternativas de marcas comerciales o formas farmacéuticas acondicionables, lo que se comunicaba al médico para su conformidad y en caso necesario, según su criterio, modificar la prescripción. En el caso de no encontrar otra opción posible, se estableció que estos medicamentos fuesen administrados en el propio centro por el enfermero.

Para la preparación de los SPD los farmacéuticos comunitarios utilizaron un protocolo adaptado del elaborado por el Consejo General de Colegios Oficiales de Farmacéuticos [5], cumpliendo la legislación vigente [6]:

- Revisión de lotes y fechas de caducidad de los medicamentos de cada paciente.

- Preparar las etiquetas individualizadas de cada paciente que se adhieren al SPD. Estas etiquetas contienen la siguiente información: código nacional, nombre del medicamento, lote, dosis, pauta y descripción de la forma y color del medicamento. Esto se realizaba tanto del medicamento que se acondicionaba como del que se administraba en el centro sociosanitario.

- Un farmacéutico rellenaba y otro revisaba los SPD. Con esto se conseguía el doble chequeo.
- Los SPD y los medicamentos no acondicionables se entregaban en el centro sociosanitario. El enfermero del centro se encargaba de organizar la administración de los medicamentos.

\section{Análisis de los datos}

En el centro sociosanitario se le asignaba un número a cada residente. En la farmacia se guardaban los datos de los pacientes en una tabla de MSExcel ${ }^{\circledR}$ de forma totalmente anónima.

Las variables que se analizaron fueron: edad, sexo, número de fármacos prescritos, criterios STOPP detectados, criterios START detectados, intervención del médico (aceptación o no aceptación de los criterios STOPP/ START detectados) y causa de no aceptación de los criterios STOPP/START por parte del médico.

Tratamiento estadístico: estadística descriptiva con MSExcel ${ }^{\circledR}$. Las variables cualitativas se expresan como porcentajes y las cuantitativas como medias.

\section{Resultados}

El centro sociosanitario tenía 25 camas. Cuando un paciente causaba baja por defunción entraba otro paciente nuevo y se le invitaba a participar en el estudio. Durante los ocho meses se produjeron 6 bajas y 6 altas siendo 31 el total de pacientes estudiados. De estos 31 pacientes, 22 fueron mujeres y 9 hombres, con una edad media de $82,9 \pm 8,1$ años.

En total se generaron 62 visitas, 31 visitas iniciales y 31 visitas de continuación. La media de fármacos prescritos en las visitas iniciales fue 5,4 $\pm 2,9$ (rango: 2-13), mientras que en las visitas de continuación la media de fármacos fue $6,0 \pm 2,9$ (rango 1-12).

\section{Criterios STOPP}

De las 62 visitas generadas, en 49 $(79,0 \%)$ visitas no se detectó ningún criterio STOPP y en $13(21,1 \%)$ sí se detectaron PPI, $10(16,1 \%)$ con un criterio y $3(4,8 \%)$ con dos.

En total se detectaron 16 PPI (tabla 1), siete de ellas correspondieron a la sección del sistema cardiovascular (B), dos a fármacos antiagregantes 
Tabla 1 Criterios STOPP detectados y motivos de rechazo

\begin{tabular}{|l|c|}
\hline Criterio STOPP & $\begin{array}{c}\text { Número } \\
\text { de casos }\end{array}$ \\
\hline $\begin{array}{l}\text { B6: Diuréticos de asa como tratamiento de primera línea de la } \\
\text { hipertensión }\end{array}$ & 3 \\
\hline $\begin{array}{l}\text { B9: Diuréticos de asa para el tratamiento de la hipertensión con } \\
\text { incontinencia urinaria }\end{array}$ & 4 \\
\hline C1: AAS en tratamiento crónico a dosis superiores a 160 mg/día & 2 \\
\hline D2: ATC como tratamiento antidepresivo de primera linea & 2 \\
\hline D5: Benzodiacepinas durante más de 4 semanas & 5 \\
\hline & 16 \\
\hline Motivo de rechazo del criterio STOPP & 6 \\
\hline Paciente controlado & 1 \\
\hline $\begin{array}{l}\text { Necesidad del medicamento porque otras alternativas no tuvieron } \\
\text { efecto. }\end{array}$ & 1 \\
\hline Intolerancia al medicamento propuesto. & $\mathbf{8}$ \\
\hline
\end{tabular}

Tabla 2 Criterios START detectados y motivos de rechazo

\begin{tabular}{|c|c|}
\hline Criterio START & $\begin{array}{l}\text { Número } \\
\text { de casos }\end{array}$ \\
\hline $\begin{array}{l}\text { A4: Antihipertensivos cuando la presión arterial sea > } 160 \mathrm{mmHg} / \\
90 \mathrm{mmHg}\end{array}$ & 3 \\
\hline A6: IECA en insuficiencia cardíaca sistólica y/o cardiopatía isquémica & 2 \\
\hline $\begin{array}{l}\text { C2: Antidepresivos no triciclicos en presencia de sintomas depresivos } \\
\text { mayores }\end{array}$ & 1 \\
\hline $\begin{array}{l}\text { C3: Inhibidores de la acetilcolinesterasa para enfermedad de } \\
\text { Alzheimer }\end{array}$ & 4 \\
\hline D1: IBP en enfermedad por reflujo gastroesofágico grave & 1 \\
\hline $\begin{array}{l}\text { D2: Suplementos de fibra en diverticulitis con antecedentes de } \\
\text { estreñimiento }\end{array}$ & 1 \\
\hline $\begin{array}{l}\text { E3: Suplementos de calcio y vitamina D con pacientes con } \\
\text { osteoporosis }\end{array}$ & 1 \\
\hline $\begin{array}{l}\text { E4: Antirresortivos o anabolizantes óseos en pacientes con } \\
\text { osteoporosis }\end{array}$ & 7 \\
\hline H2: Laxantes en pacientes que reciben opioides de forma regular & 3 \\
\hline $\begin{array}{l}\text { G1: Bloqueantes alfa-1-adrenérgicos para prostatismo sintomático } \\
\text { cuanto no se considera necesaria la prostatectomía }\end{array}$ & 1 \\
\hline $\begin{array}{l}\text { G2: Inhibidores } 5 \text {-alfa reductasa para prostatismo sintomático } \\
\text { cuando no se considera necesaria la prostatectomía. }\end{array}$ & 1 \\
\hline Total START & 25 \\
\hline \multicolumn{2}{|l|}{ Motivo de rechazo del criterio START } \\
\hline Paciente controlado & 13 \\
\hline Intolerancia al medicamento prescrito & 1 \\
\hline Diagnóstico incorrecto & 4 \\
\hline Total rechazos & 18 \\
\hline
\end{tabular}

(C) y siete al sistema nervioso central y psicotropos (D). La PPI que se detectó con mayor frecuencia fue la D5: uso de benzodiacepinas durante más de 4 semanas ( 5 casos).

De estas 16 PPI detectadas el médico aceptó un total de 8 intervenciones $(50,0 \%)$ y rechazó otras 8 (50,0\%). De los 8 casos aceptados, en 6 ocasiones se eliminó el medicamento y en 2 se cambió la dosis.

Como se observa en la tabla 1 , la causa de no aceptación más frecuente fue que el médico tenía controlado al paciente.

\section{Criterios START}

Por lo que refiere a los criterios START, se detectaron 25 prescripciones potencialmente omitidas (PPO) en $16(25,8 \%)$ de las 62 visitas totales, 9 $(14,5 \%)$ con un criterio, $6(9,7 \%)$ con dos y $1(1,6 \%)$ con 4 .

Se detectaron un total de 25 criterios START repartidos en 6 sistemas (tabla 2). Entre estos cabe destacar que 8 eran referentes a tratamientos del sistema musculoesquelético (E), siendo el criterio más repetido la falta de tratamiento con antirresortivos o anabolizantes óseos en pacientes con osteoporosis (E4) (7 casos).

De estos 25 casos con criterio START la intervención se aceptó un total de 7 casos $(28,0 \%)$ y se rechazó en 18 (72,0\%). De los 7 casos aceptados, 6 hacían referencia al inicio de tratamiento con antirresortivos o anabolizantes óseos en pacientes con osteoporosis, siendo el medicamento prescrito en todos los casos el M05BX04 (Prolia 60 mg).

De los 18 casos rechazados (tabla 2), el motivo más repetido, 13 $(72,2 \%)$ fue que el paciente estaba controlado con el tratamiento prescrito.

\section{Discusión}

Al revisar la bibliografía se encontraron estudios que analizaban la medicación con los criterios STOPPSTART en pacientes institucionalizados o en farmacia comunitaria, pero en muy pocos se mencionaba si en estos pacientes se administraba la medicación con SPD. Sin embargo, no se encontraron trabajos donde se revisara la medicación por parte del farmacéutico comunitario, se realizaran SPD y colaboraran un centro 
sociosanitario y farmacias comunitarias.

La prevalencia de criterios STOPP en nuestro estudio fue del 21\%. Es inferior que en los estudios con los que se compara y que tenían una prevalencia del 37-50\% en farmacia comunitaria $[7,8]$ y del $48-72 \%$ en centros sociosanitarios [4,7-9].

La prevalencia de criterios START en nuestro estudio fue del 32\%. Este resultado coincide con los estudios realizados en farmacia comunitaria revisados (31-33\%) [7,8]. En centros sociosanitarios la prevalencia oscila entre el 30\% y 74\% [4,9-11].

El criterio STOPP D5: uso de benzodiacepinas durante más de 4 semanas y el START falta de tratamiento con antirresortivos o anabolizantes óseos en pacientes con osteoporosis (E4) son los criterios más detectados en nuestro estudio. También son de los más frecuentes en los otros estudios que utilizan los criterios STOPP-START $[4,10,12]$.

Cuando se analizan las intervenciones del médico, se observa que los criterios STOPP se aceptan en un 50\% y los criterios START en un 28\%.

En los estudios con los que se compara la aceptación de los criterios STOPP oscila entre el 24 y el 42\%, siendo inferior al nivel de aceptación de estos criterios en nuestro estudio $[9,13,14]$.

Sin embargo, los criterios START son aceptados en más del 50\% en los estudios con los que se compara, y en nuestro estudio la aceptación es menor $[9,13,14]$.

Estos estudios donde se compara el nivel de aceptación de los criterios STOPP/START por parte de los médicos están realizados en diferentes ámbitos de aplicación y los llevan a cabo diferentes profesionales sanitarios. En ningún caso la revisión está realizada por farmacéuticos comunitarios.

Esta variabilidad encontrada en los resultados de los estudios con los que se compara nos indica que a pesar de que los criterios STOPP/START nos dan unas pautas para mejorar la farmacoterapia de los pacientes, cuando se lleva a cabo su aplicación es muy difícil eliminar la parte subjetiva del profesional sanitario que los aplica y nos demuestra la dificultad de generalizar su uso.

En referencia al nuevo funcionamiento del centro sociosanitario se han detectado muchas ventajas.
El enfermero dedica mucho menos tiempo a la preparación de la medicación ya que la mayoría llega a la residencia en el SPD. Así este puede dedicar mucho más tiempo a los cuidados propios de enfermería a los residentes. La colaboración entre los diferentes profesionales sanitarios fue totalmente voluntaria lo que facilitó en gran medida la implantación de este proyecto.

Además, gracias al SPD preparado por el farmacéutico comunitario, el personal de la residencia puede administrar la medicación más cómodamente y con menos errores. Con esta herramienta se facilita la adherencia a la medicación porque todos los tratamientos están dentro del SPD y el control de la toma de la medicación es más fácil.

Desde la implantación del nuevo proyecto se creó un protocolo de comunicación centro sociosanitario y farmacias comunitarias que mejoró la gestión de la medicación en los pacientes ingresados en la residencia. De una manera cómoda, el médico prescribe, el farmacéutico comunitario revisa el tratamiento y prepara el SPD y, finalmente, la medicación es entregada en la residencia para que el enfermero coordine su administración.

\section{Conclusiones}

- Se ha facilitado la administración de la medicación en el centro sociosanitario desde que se realizan los SPD por parte de los farmacéuticos comunitarios.

- La detección de PPI y PPO mediante la revisión de la medicación por el farmacéutico comunitario ayudó al médico a optimizar la farmacoterapia de los pacientes ingresados.

- El farmacéutico comunitario realiza, según protocolo, la revisión y preparación de la medicación dentro del equipo sanitario del centro sociosanitario.

\section{Agradecimientos}

Los autores agradecen a Silvia Sivera y al equipo de la Residencia Nuestra Señora de la Soledad de Ondara su ayuda y confianza para la realización de estudio que no hubiera sido posible llevar a cabo sin su colaboración.

\section{Referencias bibliográficas}

1. Ministerio de Sanidad, Servicios Sociales e Igualdad. Plan de calidad para el sistema nacional de salud 2010. [internet]. [consultado Sep 2017]. Disponible en: http://www.msps.es/ organizacion/sns/planCalidadSNS/informe0610/Index.html

2. Consejo general de colegios oficiales de farmacéuticos. Asistencia Farmacéutica en centros sociosanitarios: papel de la farmacia comunitaria (2017). [internet]. [consultado Sep 2017]. Disponible en: http://www. portalfarma.com/Profesionales/consejoinforma/Documents/Asistencia-farmaceutica-centros-sociosanitarios-CGCOF-febrero-2017.pdf

3. Ministerio de Sanidad, Política Social e Igualdad. Estudio EARCAS: eventos adversos en residencias y centros asistenciales sociosanitario 2011. [internet]. [consultado Sep 2017]. Disponible en: http://www.msssi.gob.es/ organizacion/sns/planCalidadSNS/ docs/EARCAS.pdf

4. Delgado-Silveira E, Montero-Errasquín B, Muñoz-García M, Vélez-Díaz Pallarés, M, Lozano-Montoya I, Sánchez-Castellano C, et al. Mejorando la prescripción de medicamentos en las personas mayores: una nueva edición de los criterios STOPP-START. Rev Esp Geriatr Gerontol. 2014; 50:89-96. doi:10.1016/j.regg.2014.10.005

5. Grupo de Trabajo de Sistemas Personalizados de Dosificación del Consejo General de Colegios Oficiales de Farmacéuticos [Internet]. Procedimiento Normalizado de Trabajo de Sistemas Personalizados de Dosificación (SPD). 2013. [Acceso 12/2/2018]. Disponible en: http://www.portalfarma.com/Profesionales/jornadasycongresos/informacion/Documents/Jornada\%20SPD/ PNT-SPD.pdf

6. Real Decreto Legislativo 1/2015, de 24 de julio, por el que se aprueba el texto refundido de la Ley de garantías y uso racional de los medicamentos y productos sanitarios. BOE; 177: 62.93563030.

7. Mud-Castello F, Mud-Castelló S, Rodriguez-Moncho MJ, Ivorra-Insa MD, Ferrándiz-Manglano ML. Detección de prescripciones potencialmente inapropiadas en pacientes ancianos: estudio descriptivo en dos farmacias comunitarias. Farmacéuticos Comunitarios. 2014; 6(2):20-26, doi:10.5672/FC.2173-9218.(2014/ Vol6).002.04

8. Barris D. Revisión de medicación según criterios STOPP/START en pacientes mayores del servicio de sistema personalizado de dosificación de medicamentos de una farmacia comunitaria. Farmacéuticos Comunitarios. 
2015 Jun 01; 7(2):31-36. doi:10.5672/ FC.2173-9218.(2015/Vol7).002.05

9. Sotoca-Momblona J, Anglada-Martínez H, Molas-Ferrer G, Fontanals-Martinez S, Rovira-Illamola M, Sebastián-Montal L. Aplicación de los nuevos criterios de prescripción inadecuada STOPP-START a pacientes geriátricos institucionalizados. FAP. 2011; 9:2-7. doi:10.1016/S21723761(11)70012-7

10. Úbeda A, Ferrandiz ML, Maicas N, Gómez C, Bonet M, Peris, JE. Potentially inappropriate prescribing in institutionalised older patients in Spain: the STOPP-START criteria compared with the Beers criteria. Pharm Pract.
2012; 10:83-91. doi:10.4321/S188636552012000200004

11. García-Gollarte F, Baleriola-Júlvez J, Ferrero-López I, Cruz-Jentoft AJ. Inappropriate drug prescription at nursing home admission. J Am Med Dir Assoc. 2012 Jan;13(1):83.e9-15. doi:10.1016/j.jamda.2011.02.009

12. O’Sullivan DP, O’Mahony D, Parsons C, Hughes C, Murphy K, Patterson $\mathrm{S}$, et al. A Prevalence Study of Potentially Inappropriate Prescribing in Irish Long-Term Care Residents. Drugs \& Aging. 2013; 30(1):39-49. doi:10.1007/s40266-012-0039-7

13. Sevilla-Sánchez D, Espaulella-Panicot J, de Andrés-Lazaro AM, To-
rres-Allezpuz R, Soldevila-Llagostera M, Codina-Jane, C. Medicación potencialmente inapropiada al ingreso en una unidad de media estancia según los criterios STOPP \& START. Rev Esp Geriatr Gerontol. 2012; 47:155-157. doi:10.1016/j. regg.2012.02.013

14. Martin-Lesende I, Mendíbil-Crespo I, Maíz-Lopez G, Gabilondo-Zelaia I, Aretxabaleta-Parra JC, Mota-Goicoechea A. Potentiality of STOPP/START criteria used in primary care to effectively change inappropriate prescribing in elderly patients. Eur Geriatr Me. 2013; 4:293-298. doi:10.1016/j. eurger.2013.06.006 